\title{
Neurobiology of sleep (Review)
}

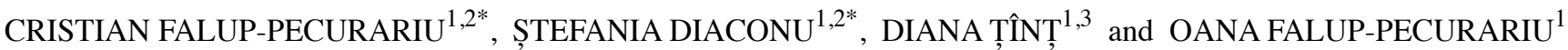 \\ ${ }^{1}$ Department of Neurology, Faculty of Medicine, Transilvania University of Brașov, 500036 Brașov; \\ ${ }^{2}$ Department of Neurology, County Emergency Clinic Hospital, 500365 Brașov; ${ }^{3}$ Clinicco Hospital, 500059 Brașov, Romania
}

Received October 12, 2020; Accepted November 10, 2020

DOI: $10.3892 /$ etm.2021.9703

\begin{abstract}
Sleep is a physiological global state composed of two different phases: Non-rapid eye movement (NREM) and rapid eye movement (REM) sleep. The control mechanisms of sleep manifest at the level of genetic, biological and cellular organization. Several brain areas, including the basal forebrain, thalamus, and hypothalamus, take part in regulating the activity of this status of life. The signals between different brain regions and those from cortical areas to periphery are conducted through various neuromediators, which are known to either promote wakefulness or sleep. Among others, serotonin, norepinephrine, histamine, hypocretin (orexin), acetylcholine, dopamine, glutamate, and gamma-aminobutyric acid are known to orchestrate the intrinsic mechanisms of sleep neurobiology. Several models that explain the transition and the continuity between wakefulness, NREM sleep and REM sleep have been proposed. All of these models include neurotransmitters as ligands in a complex reciprocal connectivity across the key-centers taking part in the regulation of sleep. Moreover, various environmental cues are integrated by a central pacemaker-located in the suprachiasmatic nucleus-which is able to connect with cortical regions and with peripheral tissues in order to promote the sleep-wake pattern.
\end{abstract}

\section{Contents}

1. Introduction

2. Anatomical regions involved in controlling sleep-wake states

3. Neuromediators in sleep/wake regulation

4. Neurobiology of wakefulness and sleep: Proposed mechanisms and models

Correspondence to: Professor Cristian Falup-Pecurariu, Department of Neurology, Faculty of Medicine, Transilvania University of Braşov, 29 Bulevardul Eroilor, 500036 Braşov, Romania

E-mail: crisfp100@yahoo.co.uk

${ }^{*}$ Contributed equally

Key words: sleep, REM sleep, NREM sleep, neurobiology, neuromediators
5. Circadian and homeostatic control of sleep

6. Conclusions

\section{Introduction}

Sleep is a reversible physiological state which involves a specific pattern of cerebral electric activity. There are two main alternating phases that compose normal sleep: Non-rapid eye movement (NREM) sleep and rapid-eye movement (REM) sleep. This article will review the main brain regions and neuromediators taking part in the regulation of sleep.

\section{Anatomical regions involved in controlling sleep-wake states}

Basal forebrain. The basal forebrain (BF) has a primary role in promoting cortical activity, mainly through cholinergic neurotransmission, as it occurs during wake and REM sleep (1). BF has various inputs from the hypothalamus and brainstem and it is also considered an important relay between the afferents from the reticular activating system to the cortex (2). Other important neurotransmitters in the basal forebrain are glutamate, vesicular glutamate transporter 3, and gamma-aminobutyric acid (GABA) (3). Because of the heterogeneity of the neurotransmitters, BF is involved in promoting both sleep and wake (mainly because of the cholinergic and GABAergic projections) (4).

The reticular activating system. The reticular activating system [formed by a group of neurons extending diffusely from the medulla to posterior hypothalamus (5)] is involved in maintaining wakefulness. The reticular formation receives afferents from various sensory systems and sends excitatory signals to the basal forebrain, thalamus and hypothalamus (6).

Thalamus. The thalamus is an important region in modulating the sleep-wake cycle, as it acts like a relay station for the glutamatergic sensory inputs to the cortex (7).

Hypothalamus. The suprachiasmatic nucleus (SCN) has various interactions with the brain, pituitary gland and autonomic nervous system (8) that will be described later in this article. The lateral hypothalamus is the only region that contains the arousal-promoting peptides hypocretins 1 and 2 . The tuberomammillary nucleus (TMN) receives excitatory inputs from 
the hypocretin-containing neurons and GABAergic inhibitory connections from the melanin-concentrating hormone $(\mathrm{MCH})$ containing neurons (9). The tonic discharge from the histaminergic neurons of the TMN induce and maintain wake, while their optogenetic-induced silence is responsive for rapid generation of slow-wave sleep (10).

The preoptic area, namely the ventrolateral preoptic area (VLPO) and the median preoptic area (MNPO), contains neurons that generate sleep by sending inhibitory projections to the arousal-promoting areas through GABA and galanin (11). These neurons discharge mostly during NREM and REM states (12). The VLPO lesions are known to reduce sleep and to alter the normal sleep architecture (13).

\section{Neuromediators in sleep/wake regulation}

Serotonin, norepinephrine (NE), histamine, hypocretins, acetylcholine (ACh), dopamine, glutamate and GABA are known mainly for having waking-promoting properties. The main structure containing neurons that express $\mathrm{NE}$ is represented by the locus coeruleus (LC), while histamine is synthesized by the TMN (14). Hypocretins (orexins) are hypothalamic neuropeptides involved in the maintenance of the wake state. Hypocretins and their precursors are regulated by the Hcrt gene, which is further modulated by several transcription factors, for instance by the early B-cell factor 2 (ebf2) (15). Hypocretins are involved in the pathophysiology of narcolepsy and have several other important physiological roles in modulating food intake and energy consumption, but may also induce negative effects, such as slower recovery after sickness due to increased amounts of NREM sleep (16). The concentration of dopamine is higher in the striatum during wakefulness and active phase (dark period) compared to its levels during slow wave sleep and resting phase (light period) (14). Glutamate, the most widespread neurotransmitter, mediates approximately $75 \%$ of the excitatory influences over the central nervous system. The levels of glutamate in several cerebral areas are the highest during arousal in waking and REM sleep states, excepting for thalamic glutamate levels, which are greater during NREM sleep (17). GABA, the main inhibitory neurotransmitter, is responsible in promoting both sleep/wake, depending on its localization and taking into account the complex interaction between other neurotransmitters, such as cholinergic and adrenergic systems $(14,18)$.

Neuromediators known as 'somnogens' are the melanin-concentrating hormone, adenosine and several cytokines (19). There are several complex interrelations between sleep modulators which are disturbed in various neurological disorders, such as Parkinson's disease or Alzheimer's disease. For instance, amyloid $\beta$ and tau proteins, which are involved in neurodegenerative processes, were found to modulate the expression of orexin A and adenosine A1 receptor in vitro and may explain the pathogenesis of certain sleep disorders in Alzheimer's disease (20).

Knowing the main roles of neuromediators has important clinical relevance, as various medications modulate the effects of neuromediators and therefore induce changes in sleep, wakefulness or circadian rhythm. For instance, anti-Parkinsonian agents such as dopamine agonists impair wake due to $\mathrm{DA}_{2}$ receptor inhibitory signaling, while levodopa and amantadine impair sleep by increasing dopaminergic activity (21).

\section{Neurobiology of wakefulness and sleep: Proposed mechanisms and models}

Wakefulness. As mentioned previously, there are several neurotransmitters that are involved in generating and sustaining the state of wake, being part of the ascending arousal system: Histamine, acetylcholine, serotonin, norepinephrine and orexin (22). From the lateral hypothalamus, there are two important pathways that ensure arousal: i) orexin neurons that stimulate the neurons in the LC, TMN and dorsal raphe nucleus (DnR)-to induce wakefulness and to stabilize the transitions between sleep and wakefulness; and ii) melanin-concentrating hormone $(\mathrm{MCH})$ with similar projections, responsible for the arousal during REM sleep (23).

Sleep. Once the wakefulness state has been suppressed, the normal human sleep cycle is composed by alternating periods of NREM and REM sleep. Each of these periods have a characteristic electroencephalographic (EEG) staging representation, but the changes in the neural activity at the transition between different stages are present long before becoming evident on EEG recording (24). The ascending arousal system is inhibited by VLPO through GABA and galanin neuro-mediation. The VLPO contains two groups of neurons: A central group projecting mostly to TMN and another diffuse one connecting with the LC and with the DnR and median raphe nuclei (23).

NREM sleep. The main brain areas that are able to generate NREM sleep are VLPO and MNPO hypothalamic regions. The neurons contained by these regions discharge preferentially during NREM sleep, being almost inactive during wake $(18,25)$. Other brain regions that generate NREM sleep through GABAergic neuromodulation are certain regions of the BF and lateral hypothalamus (25). The flip/flop switch model can be described as following. During NREM sleep, the VLPO sends inhibitory signals (through GABA and galanin) to the monoaminergic arousal systems (histaminergic neurons of TNM, cholinergic neurons of pedunculopontine tegmental nucleus, monoaminergic connections of LC and DnR), and it also inhibits the orexinergic neurons of the lateral hypothalamus. VLPO receives a mutual inhibitory projection from the arousal systems, which has low discharge during NREM sleep. The opposite events take place during wake. Orexinergic neurons act like a stabilizer for this flip-flop switch model and have an indirect inhibitory action on VLPO, as VLPO does not contain receptors for orexin $(18,26,27)$.

REM sleep. Several models that may explain REM sleep have been proposed. One proposal (a reciprocal interaction model) states that the laterodorsal tegmental nucleus and the pedunculopontine tegmental nucleus (LDT/PPT) have cholinergic excitatory projections to the reticular pontine formation (PRF), hence they promote sleep (24). The muscular atonia that is characteristic for the REM sleep period is generated by neurons located in the proximity of LC (peri locus coeruleus alpha, also known as the sublaterodorsal nucleus-SLD). 
These neurons and their connections play an important role in suppressing the muscular tone during REM. The ventromedial medulla sends inhibitory projections to the glycinergic spinal motoneurons. The muscular atonia is periodically interrupted by brief increases in muscular tone-muscle twitches-that are observed in association with rapid eye movements. These phasic muscle twitches are caused by glutamatergic excitatory outputs of the motoneurons during the activation of the central motor systems (28).

\section{Circadian and homeostatic control of sleep}

Circadian timing of sleep-wake is defined by a model that integrates two independent processes: Process $\mathrm{C}$, which denotes the variations of sleep propensity across $24 \mathrm{~h}$, and Process $\mathrm{S}$, which is related to the homeostatic sleep control (29). Several biomarkers have been found to be correlated with Process S (namely increasing during wakefulness and declining during sleep), including adenosine, prostaglandins, cytokines, brain-derived neurotropic factor and epidermal growth factor (30). The longer the duration of maintaining a state of wake exists, the higher an increase in the need for sleep. Sleep is promoted when the 'Process S', or the sleep drive, reaches the highest threshold, while the wake state begins when this factor has the lowest intensity. In this regard, NREM sleep is considered the principal marker of Process S (29).

The classical theory that explains the homeostatic control of REM/NREM alterations assumes that the need for REM sleep (REM-sleep propensity which accumulates during NREM) triggers the initiation of the REM periods. The internal circadian timing can be generated by almost every cell that has an intrinsic genetically controlled molecular clock or oscillator, but the organization and the synchronization of all of these peripheral clocks are integrated by the suprachiasmatic nucleus (31). SCN is the only oscillator that has been confirmed to receive directly the light stimuli from the retina, being able to synchronize the cues from the environment with the internal clock timing (32).

The internal molecular basis that ensures the cellular behavioral and physiological changes during the $24 \mathrm{~h}$ in mammals is governed by two transcription/translation feedback loops (TTFL), which involve, among other genes, two activating genes (CLOCK and BMALI) and two repressing genes (PER and CRY) (33). The activity of SCN neurons is increased during the REM stage and waking and decreased during the NREM stage (34). In order to generate the rhythmic shifts between sleep and wake, the SCN pacemaker integrates various types of external information, which can be photic (external light) or nonphotic, such as the rest period, locomotor activities (35), food intake or the metabolic activity (36). There is an interconnection between the mechanisms that regulate the sleep/arousal cycle, the fasting/feeding behavior and the circadian timing, all of them contributing to the maintenance of the physiological energy balance. Therefore, in order to maintain a synchrony in the circadian timing, the SCN and the periphery must be interconnected. Behavioral cues (rest/activity, locomotion, feeding) are collected by the SCN and the peripheral tissues. The periphery also influences the behavior systems and the SCN via metabolic, hormonal and autonomic pathways and all of these signals, behavioral and peripheral, are interpreted and modulated by the central pacemaker (37).

$\mathrm{SCN}$ is also involved in daily melatonin secretion rhythm control in the pineal gland. In this regard, SCN induces an inhibitory effect (via GABAergic neurons) over melatonin discharge during (subjective) daytime and a glutamatergic stimulatory action during nighttime (38).

Novel research proposals describe the role of pannexins (glycoproteins that are able to form single-membrane channels) in the control of sleep. Pannexin-1 (Panx1) hemichannels may influence sleep homeostasis by modulating the release of ATP (which further interacts with adenosine and other somnogenic cytokines) and by their vasorelaxation effects, which regulate cerebral blood flow (39). Recent evidence shows that the lateral habenula is another oscillator besides SCN, which may be able to express intrinsic circadian properties. It might receive projections from the SCN and polysynaptic information from the retina, and it might pertain to an extended circadian system (40).

\section{Conclusions}

Sleep, a vital restorative behavioral state that may be considered an undemanding physiological act, requires the perfect orchestration of many biological and chemical structures in order to be properly generated and maintained. The multiple interactions between neuromediators, brain regions and the entire organism were partially described by several proposed and demonstrated models that offer some insight into the complex mechanisms of sleep-wake regulation. Still, there are many questions left to be answered. In this regard, further studies are mandatory for a better understanding of the complex neurobiology of sleep.

\section{Acknowledgements}

Not applicable.

\section{Funding}

The present study was supported by Transilvania University of Braşov, Braşov, Romania under contract no. 8023/14.07.2017 (CFP, SD, OFP).

\section{Availability of data and materials}

All information included in this Review has documented by relevant references.

\section{Authors' contributions}

CFP made substantial contributions to the conception of the manuscript and drafted the manuscript. SD made substantial contributions to the conception of the manuscript, and drafted the manuscript. DT made substantial contributions to the conception of the manuscript, and revised it critically for important intellectual content. OFP made substantial contributions to the conception of the manuscript, and revised it critically for important intellectual content. 
All authors read and approved the final manuscript. All authors are in agreement to be accountable for all aspects of the work in ensuring that questions related to the accuracy or integrity of any part of the work are appropriately investigated and resolved.

\section{Ethics approval and consent to participate}

Not applicable.

\section{Patient consent for publication}

Not applicable.

\section{Competing interests}

The authors declare that they have no competing interests.

\section{Authors' information}

ORCID IDs: Cristian Falup-Pecurariu: 0000-0002-7394-7161; Ştefania Diaconu: 0000-0002-0251-4043; Diana Tînț: 0000-0002-6204-1364; Oana Falup-Pecurariu: 0000-0003-1972-6859.

\section{References}

1. Berntson G, Shafi R and Sarter M: Specific contributions of the basal forebrain corticopetal cholinergic system to electroencephalographic activity and sleep/waking behaviour. Eur J Neurosci 16: 2453-61, 2002.

2. Deurveilher S and Semba K: Basal forebrain regulation of cortical activity and sleep-wake states: Roles of cholinergic and non-cholinergic neurons. Sleep Biol Rhythms 9: 65-70, 2011.

3. Anaclet C, Pedersen NP, Ferrari LL, Venner A, Bass CE, Arrigoni E and Fuller PM: Basal forebrain control of wakefulness and cortical rhythms. Nat Commun 6: 8744, 2015.

4. Irmak $S$ and de Lecea L: Basal forebrain cholinergic modulation of sleep transitions. Sleep 37: 1941-51, 2014.

5. Jang $\mathrm{S}$ and Kwon $\mathrm{H}$ : The ascending reticular activating system from pontine reticular formation to the hypothalamus in the human brain: A diffusion tensor imaging study. Neurosci Lett 590: 58-61, 2015

6. Hyde $J$ and Garcia-Rill E: Ascending Projections of the RAS. In: Waking and the Reticular Activating System in Health and Disease. Garcia-Rill E (ed.) Academic Press, San Diego, pp107-128, 2015.

7. Coulon P, Budde T and Pape H: The sleep relay-the role of the thalamus in central and decentral sleep regulation. Pflugers Arch 463: 53-71, 2012.

8. Moore R: Suprachiasmatic nucleus in sleep-wake regulation. Sleep Med 8 (Suppl 3): S27-S33, 2007.

9. Yamashita $\mathrm{T}$ and Yamanaka A: Lateral hypothalamic circuits for sleep-wake control. Curr Opin Neurobiol 44: 94-100, 2017.

10. Fujita A, Bonnavion P, Wilson MH, Mickelsen LE, Bloit J, de Lecea L and Jackson AC: Hypothalamic tuberomammillary nucleus neurons: Electrophysiological diversity and essential role in arousal stability. J Neurosci 37: 9574-9592, 2017.

11. Gaus S, Strecker R, Tate B, Parker R and Saper C: Ventrolateral preoptic nucleus contains sleep-active, galaninergic neurons in multiple mammalian species. Neuroscience 115: 285-94, 2002.

12. Saper C, Cano G and Scammell T: Homeostatic, circadian, and emotional regulation of sleep. J Comp Neurol 493: 92-98, 2005.

13. Lu J, Greco M, Shiromani P and Saper C: Effect of lesions of the ventrolateral preoptic nucleus on NREM and REM sleep. J Neurosci 20: 3830-3842, 2000.

14. Oh J, Petersen C, Walsh CM, Bittencourt JC, Neylan TC and Grinberg LT: The role of co-neurotransmitters in sleep and wake regulation. Psychiatry 24: 1284-1295, 2019.
15. Sánchez-García A, Cabral-Pacheco GA, Zomosa-Signoret VC, Ortiz-López R, Camacho A, Tabera-Tarello PM, Garnica-López JA and Vidaltamayo R: Modular organization of a hypocretin gene minimal promoter. Mol Med Rep 17: 2263-2270, 2018.

16. Tanaka S, Toyoda H, Honda Y, Seki Y, Sakurai T, Honda K and Kodama T: Hypocretin/orexin prevents recovery from sickness. Biomed Rep 3: 648-650, 2015.

17. Watson $\mathrm{C}$, Lydic $\mathrm{R}$ and Baghdoyan $\mathrm{H}$ : Sleep duration varies as a function of glutamate and GABA in rat pontine reticular formation. J Neurochem 118: 571-580, 2011.

18. Gompf HS and Anaclet C: The neuroanatomy and neurochemistry of sleep-wake control. Curr Opin Physiol 15: 143-151, 2020.

19. Krueger JM, Clinton JM, Winters BD, Zielinski MR, Taishi P, Jewett KA and Davis CJ: Involvement of cytokines in slow wave sleep. Prog Brain Res 193: 39-47, 2011.

20. Liu Z, Wang F, Tang M, Zhao Y and Wang X: Amyloid $\beta$ and tau are involved in sleep disorder in Alzheimer's disease by orexin A and adenosine A(1) receptor. Int J Mol Med 43: 435-442, 2019.

21. Schneider L: Neurobiology and neuroprotective benefits of sleep. Continuum (Minneap Minn) 26: 848-870, 2020.

22. Passani M, Giannoni P, Bucherelli C, Baldi E and Blandina P: Histamine in the brain: Beyond sleep and memory. Biochem Pharmacol 73: 1113-1122, 2007.

23. Fuller P, Gooley J and Saper C: Neurobiology of the sleep-wake cycle: Sleep architecture, circadian regulation, and regulatory feedback. J Biol Rhythms 21: 482-493, 2006.

24. McCarley R: Neurobiology of REM and NREM sleep. Sleep Med 8: 302-330, 2007.

25. España R and Scammell T: Sleep neurobiology from a clinical perspective. Sleep 34: 845-858, 2011.

26. Saper C, Scammell T and Lu J: Hypothalamic regulation of sleep and circadian rhythms. Nature 437: 1257-1263, 2005.

27. Lu B and Zee P: Neurobiology of sleep. In: Sleep, an Issue of Clinics in Chest Medicine. Lee-Chiong TJ, Mohsenin V and Yaggi HK (eds.) Elsevier Health Sciences, pp309-318, 2010.

28. McKenna J, Zielinski M and McCarley R: Neurobiology of REM Sleep, NREM Sleep Homeostasis, and Gamma Band Oscillations. In: Sleep Disorders Medicine: Basic Science, Technical Considerations and Clinical Aspects. Chokroverty S (ed.) Springer, New York, pp55-68, 2017.

29. Borbély A, Daan S, Wirz-Justice A and Deboer T: The two-process model of sleep regulation: A reappraisal. J Sleep Res 25: 131-143, 2016.

30. Cuesta M, Boudreau P and Boivin D: Basic circadian timing and sleep-wake regulation. In: Sleep Disorders Medicine: Basic Science, Technical Considerations and Clinical Aspects. Chokroverty S (ed.) Springer, New York, pp55-68, 2017.

31. Schwartz W and Klerman E: Circadian neurobiology and the physiologic regulation of sleep and wakefulness. Neurol Clin 37: 475-486, 2019.

32. Dibner C, Schibler U and Albrecht U: The mammalian circadian timing system: Organization and coordination of central and peripheral clocks. Annu Rev Physiol 72: 517-549, 2010.

33. Partch C, Green C and Takahashi J: Molecular architecture of the mammalian circadian clock. Trends Cell Biol 24: 90-99, 2014.

34. Deboer T, Vansteensel M, Détári L and Meijer J: Sleep states alter activity of suprachiasmatic nucleus neurons. Nat Neurosci 6: 1086-1090, 2003.

35. Hughes A and Piggins H: Feedback actions of locomotor activity to the circadian clock. Prog Brain Res 199: 305-336, 2012.

36. Challet E: Circadian clocks, food intake, and metabolism. Prog Mol Biol Transl Sci 119: 105-135, 2013.

37. Buijs FN, León-Mercado L, Guzmán-Ruiz M, Guerrero-Vargas NN, Romo-Nava F and Buijs RM: The circadian system: A regulatory feedback network of periphery and brain. Physiology (Bethesda) 31: 170-181, 2016.

38. Perreau-Lenz S, Kalsbeek A, Garidou ML, Wortel J, van der Vliet J, van Heijningen C, Simonneaux V, Pévet P and Buijs RM: Suprachiasmatic control of melatonin synthesis in rats: Inhibitory and stimulatory mechanisms. Eur J Neurosci 17: 221-228, 2003.

39. Shestopalov V, Panchin Y, Tarasova O, Gaynullina D and Kovalzon V: Pannexins are potential new players in the regulation of cerebral homeostasis during sleep-wake cycle. Front Cell Neurosci 11: 210, 2017.

40. Baño-Otálora B and Piggins H: Contributions of the lateral habenula to circadian timekeeping. Pharmacol Biochem Behav 162: 46-54, 2017. 